Revue

Revue de l'histoire des religions

del'histoire des religions

$1 \mid 2017$

Varia

\title{
Histoire des émotions : l'heure des synthèses
}

Notes critiques

The History of Emotions: The Hour of Synthesis. Critical Notes

Bénédicte Sère

\section{(2) OpenEdition}

Journals

Édition électronique

URL : http://journals.openedition.org/rhr/8658

DOI : ERREUR PDO dans /localdata/www-bin/Core/Core/Db/Db.class.php L.34 : SQLSTATE[HYO00]

[2006] MySQL server has gone away

ISSN : 2105-2573

Éditeur

Armand Colin

Édition imprimée

Date de publication : 1 mars 2017

Pagination : 119-132

ISBN : 978-2-200-93125-4

ISSN : 0035-1423

\section{Référence électronique}

Bénédicte Sère, «Histoire des émotions : I'heure des synthèses », Revue de l'histoire des religions [En

ligne], 1| 2017, mis en ligne le 01 mars 2019, consulté le 08 janvier 2021. URL : http://

journals.openedition.org/rhr/8658; DOI : https://doi.org/10.4000/rhr.8658

Tous droits réservés 


\section{Histoire des émotions : l'heure des synthèses Notes critiques*}

En ce début d'année 2016 nous disposons de trois ouvrages, issus des meilleurs spécialistes du sujet, dans l'inscription de trois écoles historiques sur l'histoire des émotions au Moyen Âge : l'école française (Damien Boquet et Piroska Nagy), l'école italienne (Carla Casagrande et Silvana Vecchio), l'école anglo-saxonne (Barbara H. Rosenwein). Sans s'être concertés les uns les autres mais en ayant été nourris de leurs travaux réciproques, les trois courants historiques produisent leur synthèse, signe d'une maturité historiographique, scientifique et méthodologique pour une histoire des émotions désormais consacrée et reconnue dans le champ de la recherche historique actuelle.

\section{The History of Emotions: The Hour of Synthesis. Critical Notes.}

Currently, at the start of 2016, there are three works available to us from the best specialists in the subject regarding the orientations of three schools of research on the history of emotions in the Middle Ages : the French school (Damien Boquet and Piroska Nagy), the Italian school (Carla Casagrande and Silvana Vecchio), and the Anglo-Saxon school (Barbara H. Rosenwein). Without having consulted one another but having mutually influenced each other through their writings, each of these three schools has now produced a synthesis of itself, which is a sign of the historiographical, scholarly and methodological maturity of the field of the history of emotions.

* À propos des trois ouvrages suivants: Damien Boquet et Piroska Nagy, Sensible Moyen Âge. Une histoire des émotions dans l'Occident médiéval, Paris, Éd. Seuil («L'Univers historique»), 2015, 476 p., ISBN : 978-2-02-097645-9 - Carla Casagrande - Silvana Vecchio, Passioni dell'anima. Teorie e usi degli effetti nella cultura medievale, Firenze, Sismel, Edizioni del Galluzzo, 2015, 440 p., ISBN : 978-88-8450-654-2 - Barbara H. RosEnweIn, Generations of Feeling. A History of Emotions, 600-1700, Cambridge, Cambridge University Press, 368 p., 2016, ISBN : 978-1-107-09704-9. Je remercie Dominique Iogna-Prat et Patrick Henriet pour la justesse de leurs remarques sur cette note. 
De la confluence de trois traditions historiographiques sur l'histoire des émotions surgit, en cette fin d'année 2015, un puissant fleuve qui charrie la triple parution de magistrales synthèses. À la tête de l'école française, Damien Boquet et Piroska Nagy rédigent leur Sensible Moyen Âge, patiemment mûri, lentement élaboré, sûrement médité, un ouvrage de facture classique, à la portée de tous, aisément lisible et maniable. De leur côté, l'autre binôme indéfectible, Carla Casagrande et Silvana Vecchio, présente un recueil d'articles publiés depuis une dizaine d'années (dixhuit études), avec mise à jour de la bibliographie, gommage des répétitions, remaniements ponctuels et introduction générale. Cette école italienne représente la veine scolastique et théologique de l'histoire des émotions, d'Augustin à Jean Gerson. Certes, un léger pincement de cœur pourrait saisir le lecteur qui attendait une synthèse rédigée là où il retrouve des articles déjà connus, mais précisément la compilation en un seul ouvrage reste très utile. Enfin, l'ouvrage de Barbara H. Rosenwein, intitulé Generations of Feeling, précise, en sous-titre, ses bornes chronologiques de 600 à 1700, dépassant volontairement les frontières académiques entre Moyen Âge et Modernité, fidèle à la tendance anglo-saxonne dont l'auteur s'est avérée être depuis longtemps l'une des égéries pour l'histoire des émotions. Sans concertation mais avec l'estime réciproque qu'ils nourrissent les uns pour les autres, les auteurs respectifs rédigent leur synthèse en assimilant les acquis des trois traditions. Aussi la concomitance des parutions ne relève-t-elle pas tant du hasard que de la maturation scientifique : le temps des synthèses est bel et bien arrivé. Les trois ouvrages signent le couronnement d'une première saison historiographique, celle des recherches monographiques, des études de cas, des analyses micro-historiques, mais aussi celle des tâtonnements méthodologiques, des recherches d'alliances hors du champ historique - par exemple avec les neurosciences et les sciences cognitives -, celle des confrontations de résultats, celle enfin des grands programmes d'études dont EMMA (Les Émotions au Moyen Âge) reste depuis 2006 le lieu le plus fécond et la vitrine la plus utile grâce à son interface électronique. 


\section{L'HISTOIRE DES ÉMOTIONS, UN CHEMINEMENT HISTORIOGRAPHIQUE...}

La genèse de l'histoire des émotions est bien connue. Pendant longtemps, il n'y eut que mépris et dénigrement : les affects étaient rejetés et stigmatisés comme source d'inquiétude et de méfiance. Après Johan Huizinga qui peignait le tableau d'un Moyen Âge hyper-émotif, vinrent alors les autres pionniers, ceux qui ont écrit l'histoire, avec leur intuition, dans le contexte porteur de la psychologie sociale et dans l'ambiance de la Revue de synthèse: Lucien Febvre qui, dès 1941, invitait à « reconstituer la vie affective d'autrefois » mais aussi Robert Mandrou, qui appelait de ses vœux une histoire de la sensibilité, ou Georges Duby, qui se faisait peintre d'un Mâle Moyen Âge $e^{1}$ Puis, il y eut le frémissement de la fin des années 1980 et des années 1990, dans la lancée des travaux de Jean Delumeau sur la peur ${ }^{2}$, avec le séminaire de Jacques Le Goff sur le rire (1988-1991), des réflexions de Jacques Krynen ou de C. Stephen Jaeger sur l'amour des rois ${ }^{3}$, celles de Douglas Cairns sur la pudeuraîdos mais aussi les présentations méthodologiques des deux Stearns (Peter and Carol) et le concept d'emotionology ${ }^{4}$. Au début des années 2000, un bruissement plus fort se fit entendre: Piroska Nagy publiait en 2000 sa thèse de doctorat sur le Don des larmes $^{5}$;

1. L. Febvre, «La sensibilité en histoire. Comment reconstituer la vie affective d'autrefois ?», Annales d'histoire économique et sociale, 3 (1941), p. 5-20 ; R. Mandrou, «Pour une histoire de la sensibilité », Annales. Economies, Sociétés, Civilisation, 14/3 (1959), p. 581-588 ; G. Duby, Mâle Moyen Âge. De l'amour et autres essais, Paris, Flammarion, 1988.

2. J. Delumeau, La Peur en Occident, XVI $-X V I I I^{e}$ siècles, Paris, Fayard, 1978 ; Id., Le Péché et la peur. La culpabilisation en Occident (XIII'-XVIII ${ }^{e}$ siècles), Paris, Fayard, 1984 ; Id., Rassurer et protéger. Le sentiment de sécurité dans l'Occident d'autrefois, Paris, Fayard, 1989.

3. J. Krynen, L'Empire du roi. Idées et croyances politiques en France, XIII ${ }^{e}$ $X V^{e}$ siècles, Paris, Gallimard, 1993, notamment p. 458 ; Id., "Naturel. Essai sur l'argument de la nature dans la pensée politique française à la fin du Moyen Âge », Journal des Savants, avril-juin 1982, p. 169-190 ; C. S. Jaeger, «L'amour des rois : structure sociale d'une forme de sensibilité aristocratique ", Annales ESC, 3 (1991), p. 547-571.

4. D. Cairns, Aidos. The psychology and Ethics of Honour and Shame in Ancient Greek Literature, Oxford, Oxford University Press, 1993 ; P. N. Stearns et C. Z. Stearns, « Emotionology : Clarifying the History of Emotions and Emotional Standards », The American Historical Review, 90 (1985), p. 813-836.

5. P. Nagy, Le Don des larmes au Moyen Âge. Un instrument spirituel en quête d'institution, $V^{e}$-XIII' siècles, Paris, Albin Michel, 2000. 
déjà en 1997, William Reddy élaborait la notion sociologique d'emotives (ou emotional expressions à travers le langage) et en 2001, il inventait le concept de Navigation of Feeling ${ }^{6}$; enfin, Barbara H. Rosenwein rédigeait, en 2002, l'article programmatique intitule Worrying about Emotions in History dans The American Historical Review ${ }^{7}$. Indéniablement, le décollage se situe vers 2005 : Damien Boquet publie L'ordre de l'affect, mettant ainsi à la disposition du public ses conclusions doctorales soutenues trois ans auparavant ${ }^{8}$; l'essentiel des études sur les passions de Carla Casagrande et Silvana Vecchio se situe entre 2005 et 2015 ; Simo Knuuttila rédige une synthèse philosophique en 2004, Emotions in Ancient and Medieval Philosophy ${ }^{9}$; quant au célèbre Emotional Communities de B. H. Rosenwein, il date de $2006^{10}$. C'est dire que les dix années passées ont signé le succès d'un champ, sa fécondité et son dynamisme, l'intensité de sa production et l'enthousiasme de ses chercheurs. Forte de cette décennie de recherche et des acquis monographiques et analytiques, une histoire globale des émotions pouvait désormais voir le jour.

\section{L'HISTOIRE MÉDIÉVALE DES ÉMOTIONS : RECONSTITUTION D'UN RÉCIT}

Malgré leur forme différente - une histoire globale du $\mathrm{III}^{\mathrm{e}}$ au $\mathrm{XV}^{\mathrm{e}}$ siècle (D. B. et P. N.), un recueil d'articles (C. C. et S. V.), une histoire par tableau et moment-clé d'Augustin à Hobbes (B. H. R.) - les trois volumes suivent une trame narrative, à partir de laquelle l'on peut, pour l'heure, reconstituer ce qui serait l'histoire occidentale des émotions telle qu'elle s'écrit aujourd'hui, avant que d'autres

6. W. M. Reddy, «Against Constructionism. The Historical Ethnography of Emotions », Current Anthropology, 38/3 (1997), p. 327-351 ; Id., The Navigation of Feeling. A Framework for the History of Emotions, Cambridge, Cambridge University Press, 2001.

7. B. H. Rosenwein, «Worrying about Emotions in History », The American historical Review, 107 (2002), p. 821-845.

8. D. Boquet, L'ordre de l'affect au Moyen Âge. Autour de l'anthropologie affective d'Aelred de Rievaulx, Caen, CRAHM, 2005.

9. S. Knuuttila, Emotions in Ancient and Medieval Philosophy, Oxford, Oxford University Press, 2004.

10. B. H. Rosenwein, Emotional Communities in the Early Middle Ages, Ithaca, Cornell University Press, 2006. 
études et d'autres approfondissements ne viennent la compléter ou l'amender. À ce jour, voici donc la trame reconstituée de ce récit.

$\mathrm{Au}$ commencement était saint Augustin. Unanimement, en effet, Augustin est consacré comme celui qui fait entrer l'Occident médiéval dans la sphère de l'anthropologie affective, lui, le "père de l'affectivité médiévale $»^{11}$. En renversant le paradigme stoïcien selon lequel les passions étaient des maladies de l'âme, Augustin revalorise les passions et surtout, il en fait des instruments nécessaires de salut dans le contexte d'une élaboration de la doctrine chrétienne. Il est vrai, parallèlement à Augustin, persiste un front stoïcien qui s'actualise dans la tradition monastique pour qui l'apatheia, horizon de dé-passion du stoïcisme, reste l'idéal de pureté et de purification de l'âme, d'un mot la santé de l'âme. Ainsi Évagre le Pontique puis Jean Cassien insistent sur l'étude des passions de l'âme, passioni dell'anima, tout en accentuant la place du corps dans les passions. Puis vint Grégoire le Grand à qui presque tous les auteurs consacrent un épisode. Comment ne pas inscrire ses Moralia in Job dans le grand mouvement d'une histoire de la souffrance et de la douleur où l'émotion sacrificielle, dans une perspective pastorale, fixe pour les siècles à venir la liste des sept péchés capitaux et surtout assimile les passions aux vices?

Puis arrive le haut Moyen Âge. Parce que Barbara H. Rosenwein en a fait son domaine de prédilection et qu'elle excelle à se mouvoir dans les royaumes francs, les autres synthèses s'appuient sur ses travaux ainsi que sur ceux de Régine Le Jan et Nina Pancer ou encore Verena Epp ${ }^{12}$. D'autres passent littéralement sous silence le haut Moyen Âge, comme le volume italien sautant de Grégoire le Grand à Abélard et Richard de Saint-Victor au XII siècle. Barbara H. Rosenwein présente donc l'époque mérovingienne (ch. 2) et l'époque carolingienne (ch. 3) en reprenant les matériaux de sa

11. Damien Boquet et Piroska Nagy, Sensible Moyen Âge..., p. 37.

12. R. Le Jan, «Timor, amicitia, odium: les liens politiques à l'époque mérovingienne », dans W. Pohl et V. Wieser, dir., Der frühmittelalterliche Staat. Europaïsche Perspecktiven, Vienne, Österreischichen Akademie der Wissenschaften, 2009, p. 217-226 ; Ead., «Entre haine et amour du roi : quelques réflexions sur les émotions politiques à l'époque mérovingienne », dans J. Barbier, M. Cottret et L. Scordia dir., Amour et désamour du prince. Du haut Moyen Âge à la Révolution française, Paris, Kimé, 2011, p. 15-26 ; N. Pancer, Sans peur et sans vergogne. De l'honneur et des femmes aux premiers temps mérovingiens, Paris, Albin Michel, 2001 ; V. Epp, Amicitia : Zur Geschichte personaler, sozialer, politischer und geistlicher Beziehungen im frühen Mittelalter, Stuttgart, Hiersemann, 1999. 
célèbre étude Emotional Communities in the Early Middle Ages. Dans la complexité du contexte austrasien des faides et des guerres civiles puis du contexte neustrien d'une volonté de pacification sous Clothaire II au VII ${ }^{\mathrm{e}}$ siècle, B. H. Rosenwein avance le concept de «communautés émotionnelles » pour tenter de saisir les forces en présence: les communautés émotionnelles, superposables aux communautés sociales, sont appréhendées par la manière qu'elles ont d'évaluer ou de disqualifier les émotions selon des « séquences émotionnelles » et surtout des «normes émotionnelles » en vue de finalités et de valeurs rationnelles. Ainsi, l'historienne définit les normes émotionnelles de la communauté austrasienne, attachée à dire de manière presque expressionniste les émotions liées aux liens du sang et à la célébration de la famille, alors que la communauté de Neustrie adopte d'autres normes émotionnelles, et se fait plus avare d'expressivité et plus pudique dans ses émotions sinon concernant les valeurs divines et spirituelles. De la valorisation des émotions à leur stigmatisation dans les normes émotionnelles d'une communauté donnée à l'autre, l'historienne découvre un jeu politique issu d'une compétition aristocratique dont les codes de comportement évoluent au rythme des affirmations identitaires ellesmêmes. La période carolingienne est illustrée par l'échantillonnage sur la cour princière au sein de laquelle Alcuin œuvre à développer une culture particulière de l'amitié et de l'amour en vue du mot d'ordre de l'unité. En rédigeant un traité sur les vices et les vertus à l'adresse de Wido, il façonne son propre vocabulaire émotionnel.

L'époque féodale enchaîne sur les $\mathrm{XI}^{\mathrm{e}}$ et $\mathrm{XII}^{\mathrm{e}}$ siècles, dominée par l'éthique aristocratique et la culture courtoise, d'une part, par le monachisme clunisien et surtout cistercien, d'autre part. Parce que la féodalité achève de construire les liens sociaux dans des relations de personne à personne, les liens politiques tendent à s'énoncer dans le registre affectif. Toute alliance devient indissociablement politique et affective. Jongleurs et troubadours des cours aristocratiques ajoutent à cette émotion de fondement d'autres affects du monde courtois comme la joie, la tristesse, l'angoisse, la colère, la honte. Le Roman de la Rose les personnifie. La poésie les chante selon les mélodies modales associées à des valeurs morales et affectives : "La mélodie sert le texte, au sens où elle en calibre l'appréhension émotionnelle $»^{13}$.

13. Damien Boquet et Piroska Nagy, Sensible Moyen Âge..., p. 157. 
Enfin, la fin'amor exalte les émotions suscitées par l'être aimé, inaccessible ou absent - la douleur, la mélancolie, la langueurjusqu'à la joy, espoir de tout amant et fruit de l'ardente attente. Indéniablement, la littérature courtoise, et plus largement occidentale, s'avère ainsi bien plus qu'un discours sur les émotions: elle est elle-même l'émotion mise en discours ${ }^{14}$. Les $\mathrm{XI}^{\mathrm{e}}$ et $\mathrm{XII}^{\mathrm{e}}$ siècles sont également les grands siècles des mises au point monastiques d'une anthropologie affective et B. H. Rosenwein met en lumière les liens entre monastères cisterciens et mondes curiaux : les moines cisterciens n'entrent qu'adultes dans les monastères et ont bien souvent fréquenté assidûment les cours de culture courtoise (p. 141). Pensons à Aelred de Rievaulx dont la première expérience fut la fréquentation de la cour du roi David d'Écosse, dès lors son ami. Dans ces deux mondes pour lesquels l'amitié et les liens affectifs sont centraux, la compassion y est littéralement identitaire. L'historienne américaine compare ainsi les registres lexicaux des deux univers pour les confronter et les comparer. Elle conclut à une communauté de normes émotionnelles : superficiellement, la cour de Toulouse semblerait correspondre aux normes émotionnelles adoptées par les Cisterciens, même si les deux communautés ne disent pas la même chose et ne peuvent être rapprochées tant leurs valeurs et leurs buts sont différents ${ }^{15}$. Ces normes émotionnelles cisterciennes, parfaitement étudiées par Damien Boquet, métamorphosent l'appréhension spirituelle de l'homme mais aussi de la communauté monastique par le biais du concept d'affectus, c'est-à-dire par le recentrage des élans émotionnels au cœur de la relation à Dieu, et donc comme stratégie sotériologique. Originellement, rappelons-le, le monachisme n'est que méfiance envers les effusions affectives. Saint Benoît prônait la modération et condamnait les affinités électives au cloître. Or, en travaillant l'émotion en deçà de son actualisation, c'est-à-dire au niveau de l'impulsion première de la volonté, avant l'acte de consentement rationnel, les auteurs des $\mathrm{XI}^{\mathrm{e}}$ et $\mathrm{XII}^{\mathrm{e}}$ siècles, comme Anselme de Cantorbery ou Aelred de Rievaulx, font des premiers mouvements (primi motus) les vecteurs d'une conformation de l'âme à Dieu et d'une réorganisation des liens fraternels des moines au sein de la communauté. Non plus forces perturbatrices mais forces constitutives, tels sont devenus les

14. Damien Boquet et Piroska Nagy, Sensible Moyen Âge..., p. 186.

15. Barbara H. Rosenwein, Generations of Feeling..., p. 141. 
premiers mouvements des émotions. Avec Richard de Saint-Victor et la tradition victorine, la passion inscrite dans la nature humaine devient elle-même le signe de la ressemblance de l'homme à Dieu. Par nature, l'homme est passionné. Par la Passion du Christ, il trouve une légitimité à vivre ses passions. Par nature, il est tendu vers son créateur par l'élan affectif et le poids de la charité. Amor pondus meum, disait déjà Augustin. C'est à la mise en ordre des émotions pour qu'elles soient ordonnées au plan divin que s'emploient les maîtres de SaintVictor et les auteurs scolastiques du XIII ${ }^{\mathrm{e}}$ siècle. Dans le Benjamin minor de Richard de Saint-Victor, bien étudié par Silvana Vecchio, émerge une psychologie morale des émotions: "la grâce ne fait qu'ordonner les émotions (affectiones) si bien que les vertus ne sont rien d'autre que les émotions ordonnées » (Saint Bernard). Se noue alors de manière serrée l'articulation entre psychologie et théologie morale pour édifier cette anthropologie affective qui scelle le tournant sotériologique de l'histoire des émotions. Carla Casagrande et Silvana Vecchio par leur statut institutionnel respectif - chaire d'histoire des doctrines morales pour la première à l'université de Pavie et chaire d'histoire de la philosophie médiévale pour la seconde à l'université de Ferrare - se sont fait une spécialité dans l'étude et l'articulation des doctrines morales et des constructions psychologiques. La théologie de la pénitence à partir du milieu du XII ${ }^{\mathrm{e}}$ siècle leur est un terrain de prédilection pour éprouver leurs conclusions. En scrutant la honte dans le mécanisme pénitentiel, Silvana Vecchio, à partir du Benjamin minor de Richard de Saint-Victor, distingue la bonne honte, efficace et vertueuse, de la mauvaise honte liée à la crainte de déchoir socialement, celle qui inhibe l'aveu de la faute et ruine l'efficacité du sacrement de pénitence. L'auteure italienne démontre ainsi l'imbrication étroite du ressenti et de la conscience morale pour pénétrer l'intime: la honte ordonnée - ou bonne honte - est tout autant un ressenti qu'une conscience morale. C'est dire qu'à l'époque médiévale, loin de nos représentations contemporaines, le vécu émotionnel est enté sur un substrat moral. Il n'est d'authenticité du vécu (sinceritas) qu'en termes de valeur morale: "L'émotion dit le vrai de soi et d'elle-même dans la mesure où elle est liée à une valeur morale et en dernier lieu, bien entendu à Dieu $»^{16}$. Le tissage, ainsi inédit entre spiritualité, morale et psychologie, aboutit à une

16. Damien Boquet et Piroska Nagy, Sensible Moyen Âge..., p. 137. 
nouvelle anthropologie du salut, résultat indirect de la re-formatio du contexte grégorien, une anthropologie dans laquelle l'affectus devient le plus efficace instrument de con-formatio au divin. En somme, les monastères auront été les laboratoires d'une « spiritualisation de la vie sensible et émotionnelle », dont la fin'amor et l'imaginaire amoureux des cours princières furent le pendant laïque.

De ces acquis, le XIII ${ }^{\mathrm{e}}$ siècle des écoles approfondit l'étude grâce à l'essor de la pensée médicale en liant émotions et régimes de santé, ou encore émotions et dispositions physiologiques. Or, le XIII ${ }^{\mathrm{e}}$ siècle reste avant tout celui, surétudié, de Thomas d'Aquin, dont le «traité des passions », inséré au sein de la Prima Secundae de la Somme théologique demeure la référence. Vingt-six questions sont réservées au sujet. Thomas d'Aquin identifie onze passions qu'il définit comme des mouvements de l'appétit sensible de l'âme qui répondent à des sollicitations extérieures et sont accompagnés de transformations corporelles. Le psychologique est enté sur le physiologique, grâce à l'assimilation de la pensée médicale, d'une part, et œuvre à devenir une actualisation morale par la transmutation des passiones en habitus, d'autre part. L'étape thomasienne - ou plutôt albertinothomasienne si l'on suit S. V. et C. C. - est décisive d'une naturalisation de la vie affective dans l'anthropologie chrétienne.

Enfin, les derniers siècles du Moyen Âge assistent à la pénétration de cette donne affective vers le monde politique, au sein de l'élite mystique et vers les communautés laïques. En effet, le gouvernement politique par les émotions devient une réalité dont saint Louis est un acteur majeur mais aussi dont Thomas Becket a payé les frais. Le prince doit faire bon usage des émotions. Il y a une rationalité politique à intégrer la vie affective du prince et du peuple dans les modalités de gouvernement. Michel Foucault ne définissait-il pas la gouvernementalité comme l'articulation étroite entre le rapport à soi et l'exercice d'un pouvoir sur les autres ? Les émotions deviennent des événements politiques: gestes d'apaisement dans les négociations, larmes de joie, dramaturgie émotionnelle ritualisée dans les paix, les entrées royales, les cérémonies solennelles, intercession larmoyante des princesses pour obtenir une grâce, etc. Ensuite, la vie mystique plus que jamais devient le lieu d'un expressionnisme émotionnel que tout contribuait à annoncer : la féminisation du religieux, l'essor des dévotions à l'Incarnation et à la Passion, le réalisme eucharistique, 
l'allégresse du Poverello, le culte doloriste du Christ souffrant, l'auto-flagellation des laïcs pénitents dans les rues italiennes jusqu'aux auto-mutilations pénitentielles des saintes femmes telles Marie d'Oignies. Comment enfin saisir la vie émotionnelle des communautés illettrées de la fin du Moyen Âge ? Le partage social des émotions remplit la fonction d'affermir l'identité collective, de même qu'une " pastorale des émotions » (C. Casagrande) participe au projet de ré-évangélisation de l'Occident à la fin du Moyen Âge. À cette odyssée médiévale de l'affectivité, Barbara H. Rosenwein ajoute un ultime chapitre : «Hobbes' motions » où les réemplois émotionnels dans le Léviathan du $\mathrm{XVII}^{\mathrm{e}}$ siècle vise à casser la téléologie éliasienne et à rompre avec les coupures académiques entre Moyen Âge et modernité. Si l'intégration de ce XVII siècle reste peu convaincante, il ouvre néanmoins la potentialité d'une histoire des émotions à l'échelle de l'histoire universelle.

\section{Maturité AFfective du Moyen ÂGE ET MATURITÉ MÉTHODOLOGIQUE DE SES HISTORIENS}

De ce parcours affectif du millénaire médiéval, que ressort-il ? Trois points. Le premier est l'affirmation radicalement culturaliste de l'école française à laquelle se rallient, finalement, les deux autres écoles : les émotions sont des phénomènes culturels et non pas des données biologiques. D'où l'impression que l'histoire des émotions peut désormais, après les années d'expérimentation, tourner le dos à la psychologie cognitive et autres sciences neuro-biologiques pour traiter des émotions historiquement. Les émotions changent et évoluent. Leur existence reste sociale, totalement façonnée par le culturel. Il n'y a pas de surdétermination ni de soubassement biologique. L'émotion, inscrite certes dans le corps, n'existe cependant pas objectivement dans le corps, ni de manière détachée du social : les émotions demeurent des discours et des constructions sociales. Deuxièmement, les point de départ et point d'arrivée de ces trois histoires des émotions restent sensibilisés par un présupposé anti-éliasien. S'il est vrai que Norbert Elias a pu être à sa manière un historien des émotions, dissertant sur la haine, la peur, l'amour, la cruauté, la mort, ce qui lui est reproché par les médiévistes, c'est d'avoir envisagé un Moyen Âge qui pratique les émotions à l'image 
des enfants. Le grand refus, c'est l'infantilisation émotionnelle du Moyen Âge par Elias. Il n'y a pas plus de processus de civilisation qu'il n'y a d'immaturité des émotions à l'époque médiévale. En forgeant l'outil des communautés émotionnelles pour l'époque du très haut Moyen Âge, Barbara H. Rosenwein est précisément sortie du schéma évolutionniste éliasien et propose une alternative convaincante au grand récit du procès de civilisation. Troisième point : l'histoire des émotions, telle qu'elle s'écrit aujourd'hui, au terme d'une décennie de recherche, envisage le Moyen Âge sous un angle anthropologique plus que sous un angle de micro-histoire émotionnelle. Pour le dire autrement, il ne s'agit plus d'étudier telle ou telle émotion ou tel ou tel auteur isolément, mais bien, d'une certaine manière, de « reconstituer la vie affective d'autrefois » en articulant les échelles, dont le point de vue synthétique autorise désormais le jeu.

Enfin, les trois synthèses, notamment celle de D. Boquet et $\mathrm{P}$. Nagy et celle de B. H. Rosenwein, prouvent définitivement la maturité de la méthode. Si l'on pouvait encore débattre il y a dix ans sur la question des discours sociaux comme filtres des émotions vécues ou sur la question de savoir s'il fallait chercher des réalités cachées derrière les discours, des reflets derrière les miroirs des pratiques et des méta-discours derrière les textes, aujourd'hui la question ne se pose plus en ces termes. À la différence de C. C. et S. V. dont la perspective d'histoire des idées pourrait paraître assez datée, mais à qui l'on reconnaît des apports décisifs en termes de thématiques et d'axes de recherche, les auteurs français, épistémologiquement foucaldiens dès le début (surtout D. B.), ont démontré que le débat était dépassé dans la mesure où ce qu'ils pratiquent est bien une histoire sociale des concepts dans laquelle est mis au jour l'ensemble des conditions propres qui permettent à un discours d'exister. Ce qu'ils pratiquent est bien une écriture de l'histoire enracinée dans les sciences sociales plus que dans la tradition d'histoire littéraire, d'histoire des idées ou d'histoire de la philosophie. D'où le refus des oppositions frontales : il n'y a pas d'opposition entre le vécu et le ressenti, de même qu'il n'y en a pas entre le charnel et le spirituel, entre l'intime et le social, entre le biologique et la conscience, entre l'individu et son appartenance au groupe, entre le désir de l'âme et son orientation ontologique à Dieu. Les tensions qui traversent l'anthropologie sont ailleurs que dans les 
coupures binaires et les dichotomies simplistes. L'Homme tel qu'il est repensé au fil des siècles médiévaux s'est unifié et l'histoire de l'anthropologie affective nous aura convaincus de sa pertinence scientifique dans la narration de cette unification et de la sûreté de sa méthode pour revisiter les sources discursives de ce livre fait d'amitié et de larmes pour peindre le «sensible Moyen Âge».

C'est peut-être ici que l'école française se distingue de l'école italienne, plus tournée vers l'analyse philosophique des textes et les jeux d'influence entre auteurs. Carla Casagrande et Silvana Vecchio ne font-elles pas dialoguer ici Abélard et Thomas d'Aquin, et dans un autre article Augustin et Thomas ? Pour Damien Boquet et Piroska Nagy, en revanche, la production philosophique s'inscrit dans une pratique sociale. Poser ainsi la question de savoir quelle est la réalité des vécus émotionnels revient à penser l'objet historique en termes de cloisonnement stérile entre discours et réalité, là où la présente synthèse, cette histoire culturelle de l'affectivité médiévale, a convaincu du fait que l'émotion est indissociablement exprimée et ressentie, vécue et rhétorique. Pour Barbara $\mathrm{H}$. Rosenwein, la subtilité méthodologique s'accroît encore : si les discours ne correspondent pas nécessairement aux émotions vécues, ils n'en restent pas moins des modalités de la réalité émotionnelle grâce aux normes émotionnelles auxquelles adhèrent les communautés. Ce qui fait sens pour elle, ce sont les séquences émotionnelles, codifiées et normalisées, déployées dans l'espace public, plus que les émotions vécues elles-mêmes. Les séquences émotionnelles ne nous renseignent pas tant sur la manière dont les émotions sont vécues que sur le contexte dans lequel une seule et même émotion peut être vécue et exprimée différemment selon la diversité des communautés: un même symptôme émotionnel pour des significations différentes, telles les larmes de Margery Kemper, source de confort pour elle, à la différence des larmes chez les Puritains de Henry Walker au XVII ${ }^{\mathrm{e}}$ siècle, source de désagrément et de gêne. Dans son parcours d'historienne du Moyen Âge pour reprendre le titre des Journées d'études d'Auxerre qui lui avaient été consacrées, Barbara H. Rosenwein est bien passée par « la voie des émotions » pour écrire la micro-histoire sociologique qu'elle voulait écrire dès les années 1970 et qui n'a pu aboutir que parce qu'elle s'est saisie des émotions, en faisant un détour par les 
sciences cognitives ${ }^{17}$. Avec le recul du temps, la force de B. H. R. n'aura pas tant été de soutenir une thèse - au sein d'une même société, il existe un nombre limité de communautés émotionnelles, historiquement déterminées, qui s'articulent entre elles - que de penser une méthode - proposer des outils analytiques, comme les listes lexicographiques ou les «séquences », pour repérer et circonscrire ces communautés. L'utilisation des «communautés émotionnelles » donne la mesure de l'œuvre du fait qu'elle nous renseigne autant sinon plus sur les appartenances et les réseaux sociologiques que sur les émotions elles-mêmes. Toujours est-il qu'aujourd'hui, l'état des lieux d'une histoire des émotions, par l'écriture de ces synthèses, permet désormais de pointer les angles morts et les limites de l'outil. Pour le chapitre sur le $\mathrm{XVII}^{\mathrm{e}}$ siècle, le concept ne semble pas bien fonctionner. Il aurait fallu que l'auteure montre de manière plus convaincante le réemploi d'une communauté émotionnelle à l'époque de Hobbes, sa recharge pour parler comme Alphonse Dupront. Par ailleurs, il faudrait aussi valider la pertinence de l'outil pour l'ensemble de la période de la première modernité. L'invitation est lancée pour une collaboration prometteuse entre médiévistes et seiziémistes en vue d'étudier la séquence $\mathrm{XIV}^{\mathrm{e}}-\mathrm{XVI}^{\mathrm{e}}$ siècles, entre humanisme et milieux de cour, entre schismes religieux et guerres de religion, entre les théories des passions de Montaigne jusqu'au Traité des passions de l'âme de Descartes en passant par Machiavel et Vivès. De même, il faudrait confronter l'outil des « communautés émotionnelles » avec des thématiques aujourd'hui centrales dont la force et la montée en puissance interrogent, en retour, des outils plus anciens : qu'en est-il du lien avec l'espace public, avec la polémologie, avec les théories économiques autour de la pensée franciscaine, avec le gouvernement par l'enquête, etc. ? Reste encore un angle mort dans cette histoire des émotions. Ne pourrait-on pas remonter en amont de J. Huizinga et L. Febvre pour pénétrer au cœur du XIX ${ }^{\mathrm{e}}$ siècle et notamment à Jules Michelet et élargir ainsi la séquence historiographique ? Vedette des années 1970, dans les écrits de Jacques Le Goff, de Roland Barthes jusqu'à Henri de Lubac, Michelet, le narrateur de

17. De Cluny à Auxerre, par la voie des émotions. Un parcours d'historienne du Moyen Âge, B. H. Rosenwein, BUCEMA hors série, n. 5, 2013. 
l'émotionnel et de l'expression des passions, attend son historien ou ses historiens.

Pour finir, le débat peut s'ouvrir sur la question de l'imprécision sémantique qui demeure : émotions, affects, passions, sentiments, affections, sensibilités. L'école italienne revendique l'appellation passioni pour s'inscrire dans le registre christique de la Passion qui légitime la passion humaine en lui conférant sens et valeur jusqu'à en faire un instrument de salut. B. H. R., quant à elle, refuse la rigidité des acceptions sémantiques en glissant volontiers d'un vocable à un autre et en fondant les synonymies. Quant au français «émotion », il a pu avoir, dans un passé récent, mauvaise presse. Damien Boquet avait beaucoup travaillé l'affectus et recentre sa réflexion sur l'amicitia, dont la place considérable dans le livre interroge : est-ce un effet d'optique lié à la plume de l'auteur dont on sait la spécialisation ou est-ce une authentique réalité de cette omniprésence de l'amitié dans le monde médiéval, ce lien social scénarisé par l'affection ? Quoi qu'il en soit de ces complexités sémantiques, il reste que l'on pense en langue. Les trois ouvrages le prouvent. Peut-on alors parler, plutôt que d'imprécision sémantique, d'une fluidité ou d'une instabilité nécessaires à un respect des sources qui se défie des appartenances d'écoles ou des partis-pris épistémologiques, là où les taxinomies et autres nomenclatures seraient assurément plus rigidifiantes? Paradoxalement, l'instabilité ne serait alors pas manque de rigueur mais souplesse à l'écoute des sources médiévales, dont on sait la non-fixité dans les lexiques. Aussi s'explique-t-on mieux que les auteurs, Barbara H. Rosenwein notamment, revendique et assume l'imprécision sémantique.

Sans conteste, par ces trois synthèses, l'histoire des émotions prouve qu'elle a acquis ses lettres de noblesse pour exister comme champ autonome et légitime au sein de la production scientifique des historiens.

benedicte.sere@sfr.fr 